\title{
No Assistance Necessary: Arab Men's Attitudes towards Health Issues and Help-Seeking
}

\author{
Richard A. Hall \\ Open University, Milton Keynes, UK \\ Email: richhall1100@open.ac.uk
}

How to cite this paper: Hall, R. A. (2021). No Assistance Necessary: Arab Men's Attitudes towards Health Issues and HelpSeeking. Open Journal of Social Sciences, 9, 9-23.

https://doi.org/10.4236/jss.2021.911002

Received: September 29, 2021

Accepted: November 1, 2021

Published: November 4, 2021

Copyright $\odot 2021$ by author(s) and Scientific Research Publishing Inc. This work is licensed under the Creative Commons Attribution International License (CC BY 4.0).

http://creativecommons.org/licenses/by/4.0/

(c) (i) Open Access

\begin{abstract}
To what extent do gender and culture impact people's attitudes towards help-seeking? It has been well documented that gender plays a large role in determining the likelihood that an individual will look to external agencies in order to remedy issues of physical or mental health and that women tend to have more positive attitudes towards help-seeking than do men. However, this paper looks to investigate the intersection between gender and culture to understand the impact that different societies have on the formation and maintenance of attitudes towards help-seeking. In order to do this, this study utilised thematic analysis to highlight the attitudes of 3 Muslim Arab men, aged 24 - 32, in the United Arab Emirates, towards issues of mental illness and physical injury (as both are often temporary and treatable). Although much of the findings of this study were consistent with previous studies using Western participants, there were also cultural repertoires utilised which suggested that social stigma and cultural practices may further impact men's tendency to seek help from external agencies. Recognition of these differences may impact upon the promotion and delivery of healthcare in Arab countries by suggesting that providing Western treatments to Arab men, particularly for mental illness, is unlikely to be effective without cultural-specific adaptations.
\end{abstract}

\section{Keywords}

Mental-Health, Arabs, Men, Identity, Help-Seeking, Attitudes

\section{Background}

A recent World Health Organization (WHO) review of mental health claimed that men and women have notably different attitudes towards help-seeking for issues of mental health (Gough and Novikova, 2020). Indeed, studies consis- 
tently show that women are much more likely than men to seek help and engage with treatment when dealing with both physical and mental illness (Courtenay, 2000; Farrimond, 2012; Seidler et al., 2016; Sagar-Ouriaghli et al., 2019; McGraw et al., 2021). In addition to biological differences, there appear to be societal pressures and gender norms which encourage men to embody a healthy-masculine identity. Paradoxically this masculinity has at times been demonstrated to have a negative impact upon health. In the United States, for example, men are more likely than women to die from such things as cancer and heart attacks and in general suffer more chronic illness during their lifetime (Courtenay, 2000). One explanation for this is men's negative attitude towards help-seeking, which is demonstrated by the inverse-correlation between men's scores on the Conformity to Masculine Norms Inventory (CMNI) and their attitudes towards help-seeking. In addition to negative attitudes towards help-seeking, it has been found that men who scored highly on the CMNI tended to demonstrate numerous physical and mental issues such as increased blood pressure, anger, and depression as a result of "gender role stress" (Gerdes and Levant, 2018).

One explanation for the disparity between male and female attitudes are the ways in which conventional media traditionally portrays men as proponents of "stable masculinity", positioning them as invulnerable, emotionally repressed and detached from health concerns (Gough, 2006). Research by Kylie Elizabeth King and colleagues (2018) demonstrated that the use of media can be an effective tool to improve willingness to seek help. This suggests that masculine identities are malleable and subject to societal pressure. Indeed, the original hegemonic masculinities first cited by Connell $(1987,1995)$ appear to be influenced by several cultural and generational factors, further implying that masculinity is dynamic.

In some societies, progressive masculinities are being promoted more frequently in the media allowing young males to adopt non-traditional masculinity and to favour help-seeking attitudes (Gough and Novikova, 2020). However, even within these societies, attitudes are not uniform amongst sub-cultures; Asian-Americans demonstrate more negative attitudes towards help-seeking than White-Americans (Shahid et al., 2021). In other societies, such as those in the Middle East and North Africa (MENA), even with the availability of social media, stigma surrounding mental health is still at such a level that engaging openly in discourse about the subject of mental health is often difficult. Although there are claims that rates of depression, anxiety and eating disorders may be significantly higher among college students in Qatar and Lebanon compared with the USA (Kronfol et al., 2018), and that rates of depression in certain parts of United Arab Emirates society are as high as 20.03\% (Ghubach et al., 2009), negative attitudes towards mental health amongst both male and female members of Arab cultures persist. One review of Arab attitudes found that mental illness is still frequently stigmatised and attributed to such things as punish- 
ment from God, evil eye, curses, and other paranormal and supernatural phenomena (Zolezzi et al., 2018). The same review claimed that the stigma associated with mental health in Arab cultures extends beyond the individual and is likely to negatively impact their entire family's reputation. Social stigma has been demonstrated to be the biggest predictor of attitudes towards help-seeking, particularly towards Western style interventions (Al Ali et al., 2017; Velasco et al., 2020). Although negative attitudes towards mental illness appear to be prevalent across all genders in Arab culture, it is Arab men who appear to have the lowest attitudes towards help-seeking (Rayan and Jaradat, 2016).

One way that men have been shown to try and circumvent the stigma attached with mental health related help-seeking is to draw parallels with physical injuries. A leading strategy is to utilise repertoires typically reserved for physical injury (Scholz et al., 2017). This tactic appears to challenge the socially constructed dichotomy of mental and physical illness; enabling subjects to self-identify as "normal". Men are also able to focus on the active, rather than passive, aspects of help-seeking in order to place themselves in a position of power as "masculine" problem solvers. Such discourses even created a shared pleasure within the groups and seemed to aid male bonding (Gill et al., 2014). This suggests that there may be increased social pressures acting upon men to conform to masculine norms when engaging with groups of men that aren't necessarily present outside of the group setting.

Although the early work concerning hegemonic masculinity is still somewhat relevant today (Connell, 1995; Courtenay, 2000), it focuses heavily upon the hegemonic male actors and fails to fully recognise the roles that family and society play in shaping masculine ideals and identity. With this in mind, it has been suggested that labelling all forms of dominant masculinity as "hegemonic" is too simplistic and the concept of hegemonic masculinity needs to be reconstructed in light of socio-cultural factors (Connell and Messerschmidt, 2005). This is supported by Gough's (2006) suggestion that intersectionality between factors such as gender, social-class, age, race and culture, may have a larger impact upon men's discourse than gender alone.

Much of the focus of investigation, including the recent WHO report has focused upon data from Western countries (Gough and Novikova, 2020; Wei et al., 2015) and of the studies that have investigated variance in culture, many have focused on those living in America, such as Asian-American men (Luu, Leung and Nash, 2009; Shahid et al., 2021). The findings of Rayan and Jaradat (2016) indicate that Arab men, living in Arab societies will not necessarily share the attitudes of participants in those studies, nor benefit from all policy considerations outlined in the WHO report.

To address this gap, a focus group was conducted to allow participants to describe their own attitudes inside a group setting with thematic analysis utilised to identify the key themes of the participants perceptions towards health and help-seeking. The group setting also allowed individuals to develop their posi- 
tions and identities under the influence of other Arab men. The evaluation of which will illustrate the key similarities and differences between the attitudes towards issues of health and help-seeking amongst both Arab men and Western men.

\section{Method}

This study utilised a focus group format to identify and assess the attitudes of men discussing issues with mental and physical health in a group setting. Thematic analysis was chosen as the study looked to analyse the ways that participants use discourse to serve certain functions such as social positioning and power in order to maintain a healthy masculinity whilst simultaneously discussing health-related issues. Furthermore, the unstructured nature of the focus group generated rich data as participants are allowed to openly present their ideas in a natural social context (McAvoy, 2017).

\subsection{Participants}

Three Arab men from the United Arab Emirates, between the ages of 24 and 32 were invited to take part in the focus group discussion; although a larger group may have been preferable, COVID-19 restrictions in the UAE stipulated that no more than 4 people could be present. None of the participants were currently receiving treatment for any physical or mental illness and none disclosed any history of previous chronic issues. All participants partook in regular physical exercise, either CrossFit, jiu-jitsu, cycling or resistance-training. The age group was selected due to previous studies suggesting that younger generations are less likely to adopt traditional hegemonic masculine identities than their older counterparts and to be more action oriented when discussing issues of physical health (Farrimond, 2012; Gough, 2006). Written, informed consent was obtained from all participants and the study was approved by the Open University ethics panel. Participants were assigned pseudonyms to provide anonymity and all audio and transcribed data was stored on password-protected devices in accordance with the guidelines outlines by the British Psychological Society (BPS, 2014).

\subsection{Study Design}

A series of questions were developed (Appendix) to provide guidelines for the group discussion, however as the discussion was unstructured, other prompts and questions were added following topics raised by participants. A 6-phase thematic analysis was conducted (Braun and Clarke, 2006), which involved familiarisation with the data, before the identification of initial codes, revision of codes, reviewing and defining of themes and finally the generation of a report. Codes were grouped together into themes and these themes were compared across the whole data set to ensure that they were distinct (Braun and Clarke, 2006). At this stage, some codes and themes, such as the use of sporting identity to favourably position the speaker, were omitted as they did not appear as rele- 
vant to the research as other themes. Although some of the themes that emerged shared similarities with those identified in previous literature, inductive coding was utilised in order to ensure that the analysis was data-driven (Braun and Clarke, 2006). The focus of the thematic analysis was to identify the gender and cultural intersections which shape attitudes towards mental health and helpseeking.

\section{Results}

The results of the study can be broken down into two key themes. First, Arab culture appears to discourage individuals from seeking help from external sources for issues concerning mental health. This is achieved via a combination of in-group bias and stigmatisation. Second, the intersection with masculinity tends to lead to a more active, self-help, approach to dealing with health issues, which manifests primarily as a propensity towards downplaying the need for help, and then relying on themselves or close friends to remedy any issues which cannot be ignored, rather than seeking help from professional agencies.

\section{Analysis}

Although participants in this study all came from Middle Eastern backgrounds, specifically the United Arab Emirates, some of the same interpretive repertoires emerged that had previously been identified in studies conducted in the West. These included the self-helping "action man" (Farrimond, 2012) and the strong and dismissive "hegemonic" male (Courtenay, 2000). One key difference however was the overriding emphasis placed on cultural factors rather than individual factors. Wetherell and Edley (2014) did suggest that history and culture both affect, and can be affected by, discourse, however, it was not a key factor in any of the studies cited in their paper. Conversely, cultural differences were frequently mentioned by participants in this study, creating a dichotomy between the ways in which "Arabs" handle their mental health issues and the ways in which "Western people" deal with theirs. This is likely because people in modern Arab cultures are more aware of Western practices than people in the West are of Arab practices.

The participants in this study were relatively young (24 - 32) and therefore may not have the same age-related issues with physical health which were prevalent in previous studies (Farrimond, 2012). As they are active and partake in sports, their primary physical health concern was sports-related injury or underlying physical conditions that were brought on by sport and their understanding of mental health issues typically consisted of anger and depression. Throughout the discussion participants utilised both cultural and masculine identities in order to downplay any need to seek help from external agencies.

\subsection{The Impact of Arab Culture on Help-Seeking}

One of the ways in which participants in the study explained a lack of 
help-seeking with regards to issues of mental health such as depression was to utilise the cultural repertoire of "tribal Arab" vs "Western individual". Although there was no specific reference to being Arab, each of the participants demonstrated an in-group, out-group bias when utilising phrases such as "our culture" and "we", to talk about the views and behaviours within their society and used the term "European" to distance themselves from people of predominantly European descent. This Dichotomy is particularly apparent when discussing help-seeking for depression, where talking therapies are seen as a part of Western culture.

"It's not like the West, you know, in the West, as we see in movies that many people when they feel depressed, they go to a [therapist]" (Khalifa, 188).

Here the speaker distances himself and his culture away from Western therapies and implies that the requirement for therapists may be a result of Western culture. In addition, participants tended to position themselves as less individualistic and more tribal or group orientated.

"comparing us to Europe, we are a bit different, because we are more as a family together, living in the same house and we solve our problems together at home... If you are depressed from let's say, err, family issues or things like that, we don't go to the doctor, because we try to solve it always together... the lifestyle here is completely different from Europe... it's like the whole family, like all together" (Ahmed, 258).

This suggests that there is a preference in Arab men to deal with mental health issues within their community, rather than seeking help from external agencies. These cultural factors are therefore likely to inhibit help-seeking from professional mental health services, which lie outside of the tribe. In addition, attitudes towards therapy appear to be, at least partly, conceived from what is seen in movies and on TV.

In another part of the discussion, the youngest participant stepped away from this tribe-focused, suggesting that perhaps there is a lot more nuance, possibly this is greater amongst younger members of society.

"Actually now [in] our culture it's something new... everyone is feeling shy about [mental health] ...everyone wants to keep their secrets rather than share it with others... I would stay alone and get depressed alone and not tell anyone about my feelings" (Khalifa, 164).

Here the participant positions himself within the safety of the group whilst he articulates his attitudes towards mental health and help-seeking. This idea of staying alone and being depressed challenges the effectiveness of traditional practices in the modern world, where perhaps the family unit is not as closely knit today as it was in previous generations. References to culture were utilised less when discussing issues with physical health, and attitudes towards helpseeking with physical injury seemed less stigmatised. Although one member of the group seemed reluctant at first to advocate visiting a doctor, the other members challenged this and stated that it was not only acceptable to visit a doctor, 
but at times preferable.

"I would go to the doctor personally... [I would use] the physiotherapy as well to cure myself and to heal and then go back to training. I guess the doctor is the best guide" (Ahmed 59).

There appeared to be less stigma attached to utilising a physiotherapist for a sport related injury than any other form of medical help, with participants stating that they would be reluctant to take Western "chemical" medicine, instead favouring "traditional medicines". Physiotherapy aside, there was a reluctance from participants to advocate seeking help from anybody outside of their in-group or "tribe" and there similarly appeared to be a tendency to rely upon local knowledge and a preference for traditional practices. This 'tribe', however, did not appear to be exclusively limited to kin, but rather anybody who was part of their community, such as sports coaches. In addition to the stigma of seeking help for mental health, the men in the study also questioned the utility of doctors, thus seemingly justifying their preference of seeking help from friends and family.

"He [His friend] knows the injury more than the doctor, because the doctor doesn't play jiu-jitsu" (Khalifa, 42).

Whilst it is beyond the scope of this study to determine if the methods of dealing with health issues within Arab cultures are more or less effective than those utilised in Western cultures, the data suggest that Arab culture has a negative effect on attitudes towards help-seeking from professional health resources.

\subsection{The Impact of Gender Identity on Help-Seeking}

A second repertoire available to the participants was that of the "self-sufficient man", who solves his problems through a combination of strength and education. In contrast to the "tribal Arab", the enlightened and educated repertoire positions the speaker as somebody who is able to solve their own issues of physical or mental health. This is typically as a result of knowledge acquisition, a change of environment or partaking in sport to help with issues such as injury, anger, or depression. In this context, seeking medical help in addition to self-helping can be seen as the actions of an "educated" person.

"I didn't [get injured] myself, but... My friends they are well educated, most of them, but they do things like going to the doctor and starting physiotherapy and stuff to take it step by step, going to the website [and] checking [the particular] type of injury [asking themselves] what do I have to do? or what do I have to avoid? Why [did] I get injured? I'm doing CrossFit and there's a lot of technique and weightlifting" (Ahmed, 270).

Here the speaker positions himself as someone who is both masculine and strong and also manages to avoid injuries. He stated earlier in the conversation that when an individual does get injured it is because of,

"Something that he did wrong: He didn't warm up; he didn't stretch, and he didn't do the right technique" (Ahmed, 51). 
Suggesting, perhaps correctly, that if these steps are taken that the likelihood of injury is reduced. Although this approach can be beneficial, this way of thinking is likely to ascribe blame to the injured person whenever injuries do happen, even if preventative steps are followed. This line of thinking can also extend to issues of mental health, for example, a brother of one participant was advised by his Imam (religious leader) to pray more often in order to remedy his depression, rather than being advised to seek help from a mental health professional. When his depression persisted, his faith was questioned by the Imam and further prayer was prescribed.

This emphasis on self-sufficiency is similar to the "Action-Man" repertoire (Farrimond, 2012), through which the individual focuses on one's own ability to take control of the situation. Similarly, in the current study, the men appeared reluctant to rely heavily on outside help and instead looked to solve problems by educating and developing themselves.

"I like to read. So [I] read things and it will help me... going back 5 years ago I wasn't like that... [Now] I want to count on myself more than asking other people, so you have to develop yourself and err like the world is carrying on, so if you stay at the back you will be like (.) you're old fashioned, you know?" (Saif, 306).

"Sometimes, when I feel depressed or anything, I just go the gym or the jiu-jitsu training centre and err, I just forget everything I was thinking about, meeting my friends there having fun, you know, playing jiu-jitsu makes you think, you don't think anything else" (Khalifa, 110).

Although the attitudes of men in the study suggested that help-seeking from a doctor for a muscular-skeletal injury wasn't typically heavily stigmatised, it appeared that it is not socially acceptable for men to visit a doctor for issues of mental health and perhaps even less socially acceptable to suggest that another man does.

"It's in our culture to be honest... If you went to someone and you ask him this question: "why are you feeling bad, why don't you visit the doctor?" he will think that it's something bad, he will think that there's something wrong with me (Saif, 207).

However, upon broaching the subject of more vulnerable members of society one of the participants challenged the cultural ideas towards addressing mental health issues and the stigma attached.

"I'm working with child protection. Like if the kid gets abused, let's say sexually abused, err, ok he gets over it, but it's hard to get over it without a doctor, in my view. Even the parents won't take the kids to the doctor, the psycho-doctor... They are not well educated; they don't know that this is going to help them in the future" (Ahmed, 215).

Here the speaker, who works in the field of child protection, speaks of the importance of protecting weaker members of society and the importance of seeking professional help. This suggests that he believes that the stigma attached 
to help-seeking should not extend to all members of society.

As the discussion continued, there appeared more willingness to discuss the idea that the current attitudes towards mental health may need to change.

"I think physical injuries are easy to get on with after getting healed but mental health it's not, it will impact you, in my opinion, even in the future. What happened to you when you were a child will impact your personality in the future so it's something that you have to work with and something that you have to tell people about you know seeing a therapist and change this idea and the culture of the therapist and yeah, we have to, you know, educate more [people] about this thing. Not only the physical things" (Khalifa, 299).

The contrasting attitudes demonstrated in the group discussion, between help-seeking for various physical injuries and help-seeking for mental illness seem somewhat confused at times, however what does appear consistent, but to varying degrees, is the idea that seeking help from external agencies should be a last resource, especially for men, and that self-reliance and in-group practices should be utilised and exhausted first. This suggests that masculinity has a largely negative effect upon attitudes towards help-seeking.

\section{Discussion}

Analysis of the focus group discussion revealed many attitudes towards helpseeking that correspond with those highlighted by Gough and Novikova (2020). In addition to gender, the data from this study also suggest a strong intersectionality with cultural practices resulting in attitudes and subsequent behaviours towards help-seeking, which was predicted by the report (Gough and Novikova, 2020).

The men in the study appeared to focus quite heavily on the closeness of their family and community and perceived this to be a preferable means of treating such things as anger and depression, rather than seeking professional help from what they perceived to be Western therapies. A recent study of Canadian firefighters found that comparable mechanisms of utilising in-group practices such as talking with friends were preferred for dealing with the high stress roles experienced on the job. Displaying similar attitudes to participants in the current study, these Western firefighters reported that when it comes to work related issues, their colleagues "just get it" (Isaac and Buchanan, 2021). However, these attitudes towards help-seeking diverge from the attitudes of the Arab men in the current study when it comes to non-work-related issues, such as marriage and mental health. In these non-work-related circumstances, the study's data suggests that firefighters had a preference towards seeking professional help, whereas participants in the current study remained reluctant to utilise out-group specialists themselves, or to recommend their use to other men. One explanation for this is that these informal solutions tend to be brief and less stigmatised for Arabs (Al-Krenawi et al., 2009). The participants in the current study frequently indicated that either help-seeking oneself or suggesting that someone else seek 
help is socially taboo. In addition to the negative effects of stigma upon attitudes towards help-seeking, studies have shown that loneliness and a perceived social support were good predictors of levels of depression in studies across the UK and USA (Wang et al., 2018) and that creating strong social connections help to reduce levels of depression and anxiety (Hari, 2018). Studies which revealed high levels of depression and anxiety in UAE also found that promoting family support and not living alone to be especially helpful for combatting issues of mental health (Ghubach et al., 2009). Therefore, it is unlikely that any single factor is responsible for the attitudes of Arab men towards help-seeking, rather, the negative effects of stigma and the positive impact of in-group support are likely to combine in order to deter out-group help-seeking for all but the most serious mental health issues.

Although stigma towards help-seeking for mental health issues in Arab culture was demonstrated in the current study, participants made no reference to a belief that the cause of such things as depression or anxiety lie in the supernatural; punishment from God or evil eye, which has been heavily linked to Arab culture and attitudes in previous studies (Al Ali et al., 2017; Zolezzi et al., 2018). In addition, participants demonstrated an understanding of the need for professional help with more serious issues of mental health and expressed a desire for a change in culture. These shifts may be indicative of similar generational shifts in masculinity and attitudes to those which have been witnessed in the West (Anderson, 2017).

In addition to the cultural aspects of attitudes towards mental health and help-seeking in general, participants in the study also demonstrated gender specific attitudes, similar to those found in previous studies, particularly a propensity towards self-reliance and problem solving (Gough and Novikova, 2020; Gill et al., 2014). The men in the current study spoke of a preference to solve problems with depression and anxiety by exercising or partaking in sports and demonstrated positive attitudes towards prioritising their own well being and the need to adopt health-promoting behaviours. This emphasis upon healthy eating, exercise and sufficient rest echoes the data presented by the WHO report (Gough and Novikova, 2020) and may suggest a masculinity far removed from the original idea of hegemony postulated by Connell $(1987,1995)$, indicating that attitudes towards what constitutes hegemonic masculinity are changing and are likely to be subject to external influences.

All methods of psychological investigation are open to researcher bias, and this study is no different; when it comes to qualitative methods as data are not coded in an epistemological vacuum. It follows that although every attempt was made to set aside any pre-conceptions that I may have formed following my exposure to Arab culture, it is possible that some of my own opinions impacted upon the coding and thematic analysis. Due to the small sample size, the data from this study are not generalizable to all Arab societies, but rather act as a launch pad for further investigation. The focus group was conducted in English 
which is a second language for all three participants and research suggests that the use of one's second language has the potential to impact upon one's attitudes and personality (O'Brien et al., 2005) as well as the intensity to which one sticks to cultural ideals (Itzhak et al., 2017). Finally, as the participants in this study did not declare any form of chronic illness themselves it is impossible to extrapolate the attitudes of those suffering from such afflictions, however, as the study's aim was to understand the impact of culture and gender on attitudes towards help-seeking, this is not necessary. What has been demonstrated by this study is that culture plays a large role in the forming and maintaining of attitudes towards help-seeking. Furthermore, the intersection between culture and gender appears to have a greater effect than culture or gender alone.

\section{Conclusion and Recommendations}

The Arab men in this study demonstrated a reluctance to seek help from professional institutions, which they appeared to view as aspects of Western culture. Instead, participants demonstrated a preference towards one of two alternative options. The first is to deal with issues themselves via such processes as partaking in exercise, changing scenery or via the acquisition of knowledge. The second is to trust in-group members such as family or community for advice and support.

The self-reliance suggested by the first of these two options marries well with the findings of previous research into male help-seeking behaviour among Western males and therefore would likely benefit from the same policy considerations outlined by Gough and Novikova (2020: p. 34), particularly those that include tackling the causes of isolation and those promoting strength-based approaches which require active, rather than passive participation.

The data from the current study digress slightly from the WHO report, however, when focus is shifted towards in-group processes. The attitudes demonstrated during this study suggest that an availability of external sources alone will be insufficient to penetrate Arab culture, and that both stigma and a bias towards traditional remedies will remain.

Eastern cultures tend to be more collectivist than Western cultures (Triandis, 1998) and it is possible that by including the family or community in health care promotion that attitudes can be changed from within, targeting parents as well as children and young adults may be an effective method to changing the narrative associated with mental health in Arab culture. The use of community engagement as well as in-group actors, such as friends and teachers has been shown to be effective in improving attitudes of adolescents (Velasco et al., 2020), and it is hoped that these attitudes will persist into adulthood.

Another method that could be utilised in conjunction with community outreach and may be uniquely successful in the region is that of top-down information campaigns. The WHO (Gough and Novikova, 2020) recommend engaging in health-focused programmes and websites in order to change attitudes in Eng- 
lish and Russian speaking countries, however Arab nations, such as UAE and Saudi Arabia appear to trust their government and national media for information more than do people in the UK, Russia or USA (Edelman Trust Barometer, 2021) so may benefit from overt government intervention more than people in Western societies. Raising awareness of the benefits of treatments and improving knowledge about treatment availability this way can help to improve attitudes towards help-seeking and therefore long-term health outcomes (King et al., 2018). It follows that the implementation of national mental health policies may have a greater impact on attitudes and help-seeking of people in Arab and Asian regions than it would in Western nations. The effectiveness of such approaches is therefore a good stepping off point for further investigation.

The small size of this study means that results cannot be generalised, however it does demonstrate that the intersection between gender and culture appears to have an impact upon the attitudes of Arab men towards help-seeking and should be investigated further before mental health related policies are implemented.

\section{Conflicts of Interest}

The author declares no conflicts of interest regarding the publication of this paper.

\section{References}

Al Ali, N., Alqurneh, M., Dalky, H., \& Al-Omari, H. (2017). Factors Affecting Help-Seeking Attitudes Regarding Mental Health Services among Attendance of Primary Health Care Centers in Jordan. International Journal of Mental Health, 46, 38-51. https://doi.org/10.1080/00207411.2016.1264039

Al-Krenawi, A., Graham, J. R., Al-Bedah, E. A. et al. (2009). Cross-National Comparison of Middle Eastern University Students: Help-Seeking Behaviors, Attitudes toward Helping Professionals, and Cultural Beliefs about Mental Health Problems. Community Mental Health Journal, 45, 26. https://doi.org/10.1007/s10597-008-9175-2

Anderson, E. (2017). Generational Masculinities. Journal of Gender Studies, 27, 243-247.

Braun, V., \& Clarke, V. (2006). Using Thematic Analysis in Psychology. Qualitative Research in Psychology, 3, 77-101. https://doi.org/10.1191/1478088706qp063oa

British Psychological Society (BPS) (2014). Code of Human Research Ethics. https://www.bps.org.uk/sites/www.bps.org.uk/files/Policy/Policy\%20-\%20Files/BPS\%2 0Code\%20of\%20Human\%20Research\%20Ethics.pdf

Connell, R. W. (1987). Gender and Power (pp. 279-304). Polity.

Connell, R. W. (1995). Masculinities. University of California Press.

Connell, R. W., \& Messerschmidt, J. T. (2005). Hegemonic Masculinity: Rethinking the Concept. Gender and Society, 19, 829-859. https://doi.org/10.1177/0891243205278639

Courtenay, W. (2000). Constructions of Masculinity and Their Influence on Men's Well-Being: A Theory of Gender and Health. Social Science \& Medicine, 50, 1385-1401. https://doi.org/10.1016/S0277-9536(99)00390-1

Edelman Trust Barometer (2021). https://www.edelman.com/sites/g/files/aatuss191/files/2021-03/2021\%20Edelman\%20T rust $\% 20$ Barometer.pdf 
Farrimond, H. (2012). Beyond the Caveman: Rethinking Masculinity in Relation to Men's Help-Seeking. Health, 16, 208-225. https://doi.org/10.1177/1363459311403943

Gerdes, Z., \& Levant, R. (2018). Complex Relationships among Masculine Norms and Health/Well-Being Outcomes: Correlation Patterns of the Conformity to Masculine Norms Inventory Subscales. American Journal of Men's Health, 12, 229-240. https://doi.org/10.1177/1557988317745910

Ghubach, R., El-Rufaie, O., Zoubeidi, T., Sabri, S., Yousif, S., \& Moselhy, H. F. (2009). Subjective Life Satisfaction and Mental Disorders among Older Adults in UAE in General Population. International Journal of Geriatric Psychiatry, 25, 458-465. https://doi.org/10.1002/gps.2360

Gill, P., Teese, R., \& Sonn, C. (2014). Masculine Identity Negotiation in Everyday Australian Life: An Ethno-Discursive Study in a Gym Setting. International Journal of Men's Health, 13, 42-59. https://doi.org/10.3149/jmh.1301.42

Gough, B. (2006). Try to Be Healthy, But Don't Forgo Your Masculinity: Deconstructing Men's Health Discourse in the Media. Social Science \& Medicine, 63, 2476-2488. https://doi.org/10.1016/j.socscimed.2006.06.004

Gough, B., \& Novikova, I. (2020). Mental Health, Men and Culture: How Do Sociocultural Constructions of Masculinities Relate to Men's Mental Health Help-Seeking Behaviour in the WHO European Region? Technical Report, WHO.

https://eprints.leedsbeckett.ac.uk/id/eprint/7159

Hari, J. (2018). Lost Connections: Why You're Depressed and How to Find Hope. Bloomsbury Publishing.

Isaac, G., \& Buchanan, M. (2021). Extinguishing Stigma among Firefighters: An Examination of Stress, Social Support, and Help-Seeking Attitudes. Psychology, 12, 349-373. https://doi.org/10.4236/psych.2021.123023

Itzhak, I., Vingron, N., Baum, S., \& Titone, D. (2017). Bilingualism in the Real World: How Proficiency, Emotion, and Personality in a Second Language Impact Communication in Clinical and Legal Settings. Translational Issues in Psychological Science, 3, 48-65. https://doi.org/10.1037/tps0000103

King, K. E., Schlichthorst, M., Spittal, M. J., Phelps, A., \& Pirkis, J. (2018). Can a Documentary Increase Help-Seeking Intentions in Men? A Randomised Controlled Trial. Journal of Epidemiology \& Community Health, 72, 92-98. https://doi.org/10.1136/jech-2017-209502

Kronfol, Z., Khalifa, B., Khoury, B. et al. (2018). Selected Psychiatric Problems among College Students in Two Arab Countries: Comparison with the USA. BMC Psychiatry, 18, 147. https://doi.org/10.1186/s12888-018-1718-7

Luu, T. D., Leung, P., \& Nash, S. G. (2009). Help-Seeking Attitudes among Vietnamese Americans: The Impact of Acculturation, Cultural Barriers, and Spiritual Beliefs. Social Work in Mental Health, 7, 476-493. https://doi.org/10.1080/15332980802467456

McAvoy, J. (2017). Why Methods Matter. In J. McAvoy, \& N. Brace (Eds.), Investigating Methods (pp. 9-46). The Open University.

McGraw, J., White, K. M., \& Russell-Bennett, R. (2021). Masculinity and Men's Health Service Use across Four Social Generations: Findings from Australia's Ten to Men Study. SSM-Population Health, 15, Article ID: 100838.

https://doi.org/10.1016/j.ssmph.2021.100838

O’Brien, R., Hunt, K., \& Hart, G. (2005). "It's Caveman Stuff, But That Is to a Certain Extent How Guys Still Operate”: Men's Accounts of Masculinity and Help Seeking. Social Science and Medicine, 61, 503-516. https://doi.org/10.1016/j.socscimed.2004.12.008 
Rayan, A., \& Jaradat, A. (2016). Stigma of Mental Illness and Attitudes toward Psychological Help-Seeking in Jordanian University Students. Research in Psychology and Behavioural Sciences, 4, 7-14.

Sagar-Ouriaghli, I., Godfrey, E., Bridge, L., Meade, L., \& Brown, J. S. L. (2019). Improving Mental Health Service Utilization among Men: A Systematic Review and Synthesis of Behavior Change Techniques within Interventions Targeting Help-Seeking. American Journal of Men's Health, 13, 1-8. https://doi.org/10.1177/1557988319857009

Scholz, B., Crabb, S., \& Wittert, G. A. (2017). “Males Don't Wanna Bring Anything Up to Their Doctor”: Men's Discourses of Depression. Qualitative Health Research, 27, 727-737. https://doi.org/10.1177/1049732316640294

Seidler, Z. E., Dawes, A. J., Rice, S. M., Oliffe, J. L., \& Dhillon, H. M. (2016). The Role of Masculinity in Men's Help-Seeking for Depression: A Systematic Review. Clinical Psychology Review, 49, 106-118. https://doi.org/10.1016/j.cpr.2016.09.002

Shahid, M., Weiss, N. H., Stoner, G., \& Dewsbury, B. (2021). Asian Americans' Mental Health Help-Seeking Attitudes: The Relative and Unique Roles of Cultural Values and Ethnic Identity. Asian American Journal of Psychology, 12, 138-146.

https://doi.org/10.1037/aap0000230

Triandis, H. C. (1998). Individualism \& Collectivism. Westview Press. https://doi.org/10.1037/t01556-000

Velasco, A., Cruz, I. S. S., Billings, J. et al. (2020). What Are the Barriers, Facilitators and Interventions Targeting Help-Seeking Behaviours for Common Mental Health Problems in Adolescents? A Systematic Review. BMC Psychiatry, 20, 293. https://doi.org/10.1186/s12888-020-02659-0

Wang, J., Mann, F., Lloyd-Evans, B. et al. (2018). Associations between Loneliness and Perceived Social Support and Outcomes of Mental Health Problems: A Systematic Review. BMC Psychiatry, 18, 156. https://doi.org/10.1186/s12888-018-1736-5

Wei, Y., McGrath, P. J., Hayden, J. et al. (2015). Mental Health Literacy Measures Evaluating Knowledge, Attitudes and Help-Seeking: A Scoping Review. BMC Psychiatry, 15, 291. https://doi.org/10.1186/s12888-015-0681-9

Wetherell, M., \& Edley, N. (2014). A Discursive Psychological Framework for Analyzing Men and Masculinities. Psychology of Men \& Masculinity, 15, 355-364. https://doi.org/10.1037/a0037148

Zolezzi, M., Alamri, M., Shaar, S., \& Rainkie, D. (2018). Stigma Associated with Mental Illness and Its Treatment in the Arab Culture: A Systematic Review. International Journal of Social Psychiatry, 64, 597-609. https://doi.org/10.1177/0020764018789200 


\section{Appendix: Focus Group Questions}

What are your general attitudes towards health?

Do your attitudes towards the physical and mental aspects of health differ?

How have you or in the past or how do you think that you would respond to any issues concerning your physical health?

How have you or in the past or how do you think that you would respond to any issues concerning your mental health?

How do you think that your responses would be viewed by other people?

Do you think that your attitudes are similar to other people in your society?

How comfortable would you be with seeking help from a doctor or therapist? Do you think that your attitudes have changed over time? 